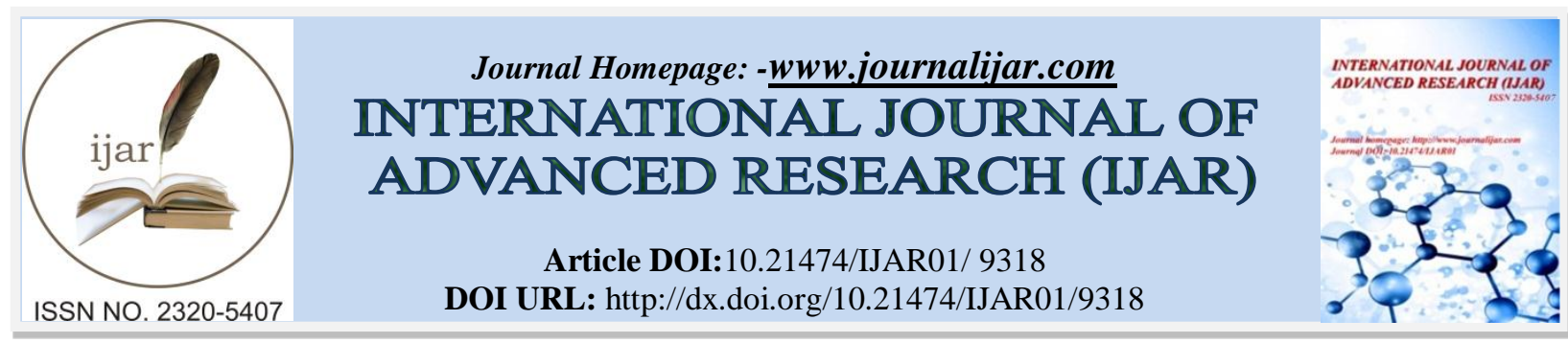

RESEARCH ARTICLE

\title{
POST TRAUMATIC RETROCLIVAL EPIDURAL HEMATOMA IN A YOUNG ADULT.
}

M Benzalim, L berghalout, S alj, M Ouali Idrissi and N Idrissi Gannouni. Service de radiologie CHU Mohammed VI, Marrakech Université CADI AYYAD.

\section{Manuscript Info}

\section{Manuscript History}

Received: 02 May 2019

Final Accepted: 04 June 2019

Published: July 2019

Key words:-

hematoma ; epidural ; retroclival ; adult ; imagery.

\begin{abstract}
Introduction: Post-traumatic hematoma of the posterior fossa are rare and account for only $3 \%$ of all cerebral post-traumatic hematoma. They are common along the cerebellar convexity while retroclival location is rare with less than 30 cases reported in the literature. Most previously reported cases occurred in children, with only a small number of cases involving adult patients. Among these adult cases, only three had an associated fracture of the occipital condyle.

Objectives:To report a rare case of epidural hematoma of retroclival seat in a young adult, review the pathophysiology and mechanisms of occurrence of these hematomas.

Observation : We related the case of a young man of 25 years without disease history, with a following an AVP retroclival epidural hematoma associated with a fracture of the clivus extended to the right occipital condyle diagnosed on a brain CT conservatively treated.

Conclusion:his type of hematoma is not exclusive to the pediatric population, and although there are few reported cases, it is possible that this entity is often under diagnosed and must involve the exploration of the CCO for eliminate ligament underlying instability.
\end{abstract}

Copy Right, IJAR, 2019,. All rights reserved.

\section{Introduction:-}

Les hématomes extra duraux sont les lésions post traumatiques les plus fréquemment rencontrées dans la fosse cérébrale postérieure [1]. Cependant, ceux de localisation retroclivale sont exceptionnellement rares [1, 2, 3]. Cette entité a été décrite la première fois en 1941 par Coleman et Thomson [2], et depuis lors, moins de 30 cas d'hématome épidural retroclival post traumatique ont été rapportés dans la littérature [4], intéressant presque exclusivement la population pédiatrique [3, 4]. Le cas que nous rapportons est l'un des rares cas rapportés chez l'adulte. Cliniquement, l'hématome extradural retroclival est habituellement silencieux et lent, mais la détérioration peut être soudaine et rapidement fatale par l'installation d'une hydrocéphalie obstructive en l'absence de traitement rapide [2].

\section{Observation :}

Un homme de 25 ans, victime d'un accident violent de la voie publique ; motocycliste heurté par une voiture, occasionnant chez lui une fracture fermée du fémur droit pour laquelle il a été admis aux urgences médicochirurgicales. A l'admission, le patient était conscient avec un GCS estimé à 15, l'interrogatoire ne retrouve pas de notion de perte de connaissance initiale ou de vomissement. Le patient était stable sur le plan respiratoire et hémodynamique avec une TA à 120/70 mmHg, sans trouble de conscience ni déficit neurologique notable. Un bilan 
radiologique et biologique a été réalisé, fait de radiographies standard du fémur de face et de profil, du bassin de face, du thorax de face, échographie abdominale, une numération formule et groupage sanguin. Au terme de ce bilan, le patient fut hospitalisé au service de traumatologie pour prise en charge de sa fracture fermée et déplacée du fémur droit, et mis sous traction avant le traitement chirurgical.

24 heures après son hospitalisation, le patient présente des troubles de consciences avec détresse respiratoire. Une TDM cérébrale fut alors réalisée objectivant la présence en retroclival d'une collection extra durale spontanément hyperdense mesurant $5 \mathrm{~mm}$ d'épaisseur maximale et étendu sur $24 \mathrm{~mm}$ (figure 1 et 2), non rehaussé par le produit de contraste, associé à une fracture peu déplacée du clivus (figure 4), étendue au condyle occipital droit, en faveur d'un hématome extra dural. Cet hématome affleure la face antérieure de la protubérance et entre en étroit rapport avec le tronc basilaire (figure3). Un angioscanner cérébral a été alors effectué n'objectivant pas d'anomalie vasculaire vertébro-basilaire associée (figure3).

Le patient a été mis sous surveillance en réanimation chirurgicale, opéré pour sa fracture du fémur. L'évolution a été marquée par une amélioration clinique dès le troisième jour, avec disparition des céphalées et rétablissement de son état de conscience. Le contrôle scanographique à deux semaines a objectivé la régression de l'HED retroclival sans hématome résiduel.

\section{Discussion:-}

Les traumatismes constituent la cause la plus fréquente des hématomes extraduraux [2]. La plupart des cas sont associés à des fractures du crâne et contrairement aux hématomes périduraux supratentoriels, qui sont dus principalement à des lésions artérielles, la plupart des hématomes extraduraux de la FCP sont dus à une atteinte des sinus veineux $[2,5]$.

La physiopathologie de la formation d'un hématome épidural retroclival n'est pas encore bien élucidée [2, 3]. Il a été suggéré que les caractéristiques particulières de la jonction cranio-vertébrale prédisposent à la formation de ces hématomes puisqu'on note une stabilité précaire de la jonction cranio-vertébrale essentiellement pendant l'enfance [3] car les condyles occipitaux sont relativement plus petits et l'articulation atlanto-occipitale est orientée plus horizontalement. En outre, l'atteinte et la dislocation du tissu ligamentaire reliant le processus odontoïde à la base du crâne pourrait être impliqué dans cette physiopathologie. L'allongement et la dislocation de la membrane tectoriale, du ligament longitudinal postérieur et du ligament cruciforme pourrait perturber les structures vasculaires locales au niveau de la charnière cervico-occipitale (plexus veineux basilaire, branche méningée dorsale du tronc meningohypophysaire) conduisant à une accumulation de sang en retroclival [6, 7].

Les mécanismes en hyperflexion ou en hyperextension craniocervicale ont été rapportés $[2,6,7]$. Certains auteurs ont également rapporté la notion d'instabilité ligamentaire au niveau de la $\mathrm{CCO}$ et qui doit être suspectée dans un tel cas [2].

La TDM est la méthode d'imagerie à réaliser en première intention face à un traumatisme cérébral, mais les artéfacts de durcissement peuvent masquer ces hématomes [8]. L'IRM est l'outil de diagnostic le plus sensible pour diagnostiquer ces lésions [2]; elle permet de palier aux artéfacts de durcissement rencontrés au scanner, avec une grande sensibilité grâce à la séquence $\mathrm{T} 2 *$ permettant la mise en évidence des produits de dégradation de l'hémoglobine, tels que la désoxyhémoglobine qui est responsable d'un hyposignal par effet de susceptibilité magnétique.

Toutefois, les reconstructions multi planaires notamment sagittales et l'angioscanner peuvent augmenter la sensibilité de la TDM pour la détection des hématomes de la FCP de siège retroclival et ajouter d'autres informations tel qu'une éventuelle fracture clivale ou une lésion vasculaire associée, comme il est le cas dans cette observation.

La taille de l'hématome selon la littérature n'a aucune corrélation avec la gravité des symptômes et des signes cliniques $[3,9]$. De même que l'état de conscience initial jugé par le score de Glasgow, ne permet pas de prévoir le pronostic des patients $[3,7,9]$.

Une revue de la littérature [2, 3, 4] montre que le traitement conservateur est généralement adopté en première ligne, seuls les cas avec compression critique des éléments de la FCP doivent bénéficier d'une évacuation chirurgicale de l'hématome du fait du risque hémorragique élevé. 


\section{Conclusion:-}

L'hématome épidural retroclival post traumatique n'est pas exclusif à la population pédiatrique, et bien qu'il y ait peu de cas rapportés, il est possible que cette entité est souvent sous diagnostiquée et doit impliquer l'exploration de la CCO à fin d'éliminer une instabilité ligamentaire sous-jacente.

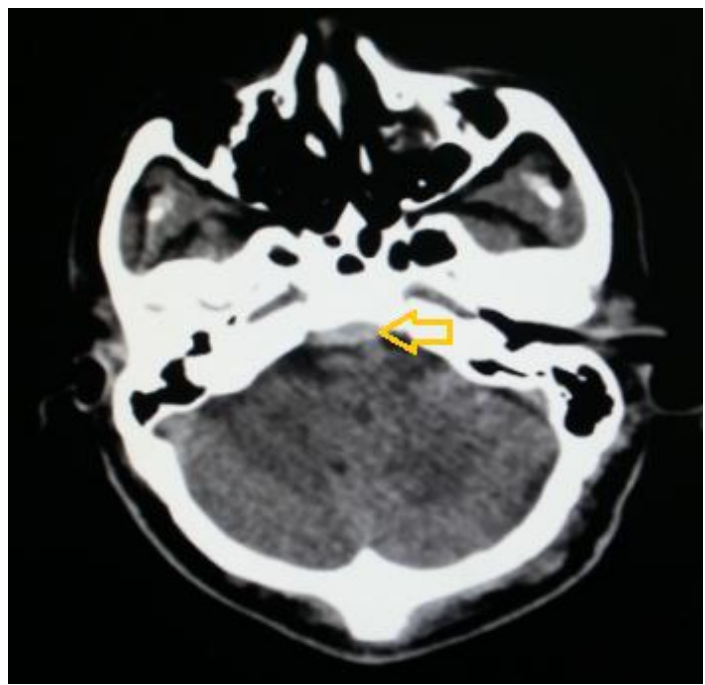

Figure 1:coupe scanographique axiale sans injection de PDC, objectivant une collection retroclivale en lentille biconvexe, spontanément hyperdense en rapport avec un hématome extra dural.

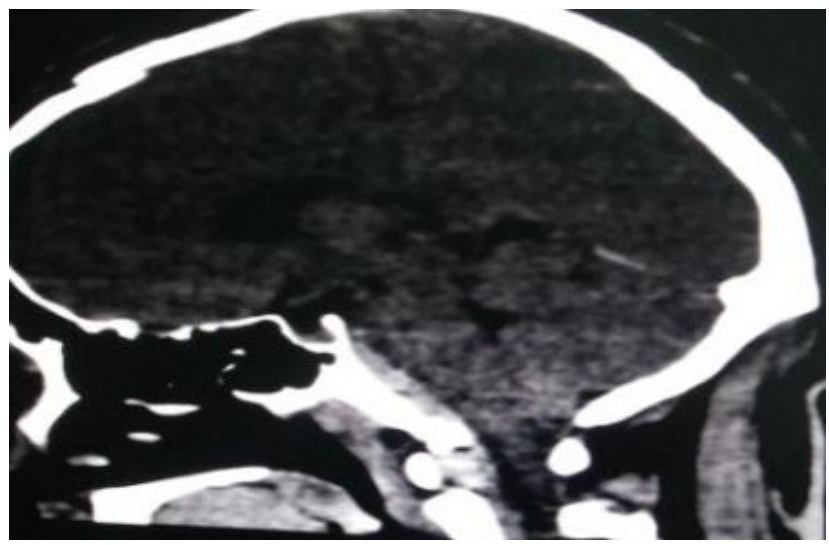

Figure 2:-coupe scanographique sagittale sans injection de PDC, objectivant l'hématome extra dural retroclival.

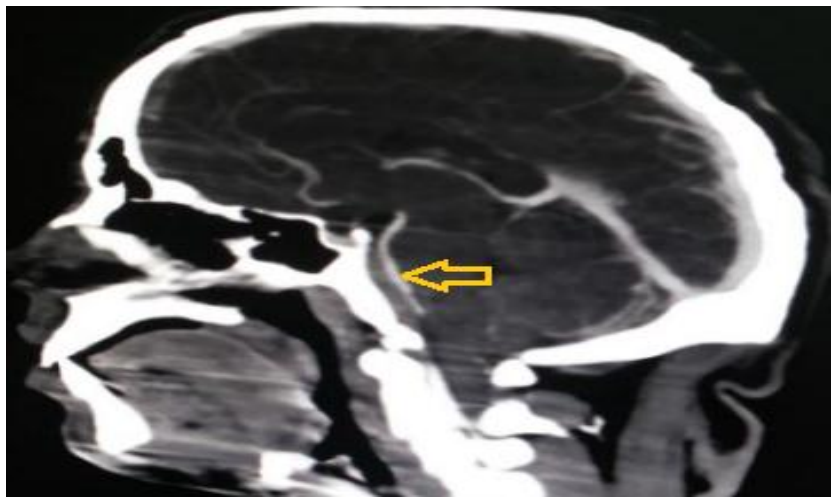

Figure 3:-coupe scanographique sagittale après injection de PDC, mode MIP, objectivant le rapport de l'hématome extra dural retroclival avec le tronc basilaire qui est normalement opacifié, de calibre normal. 

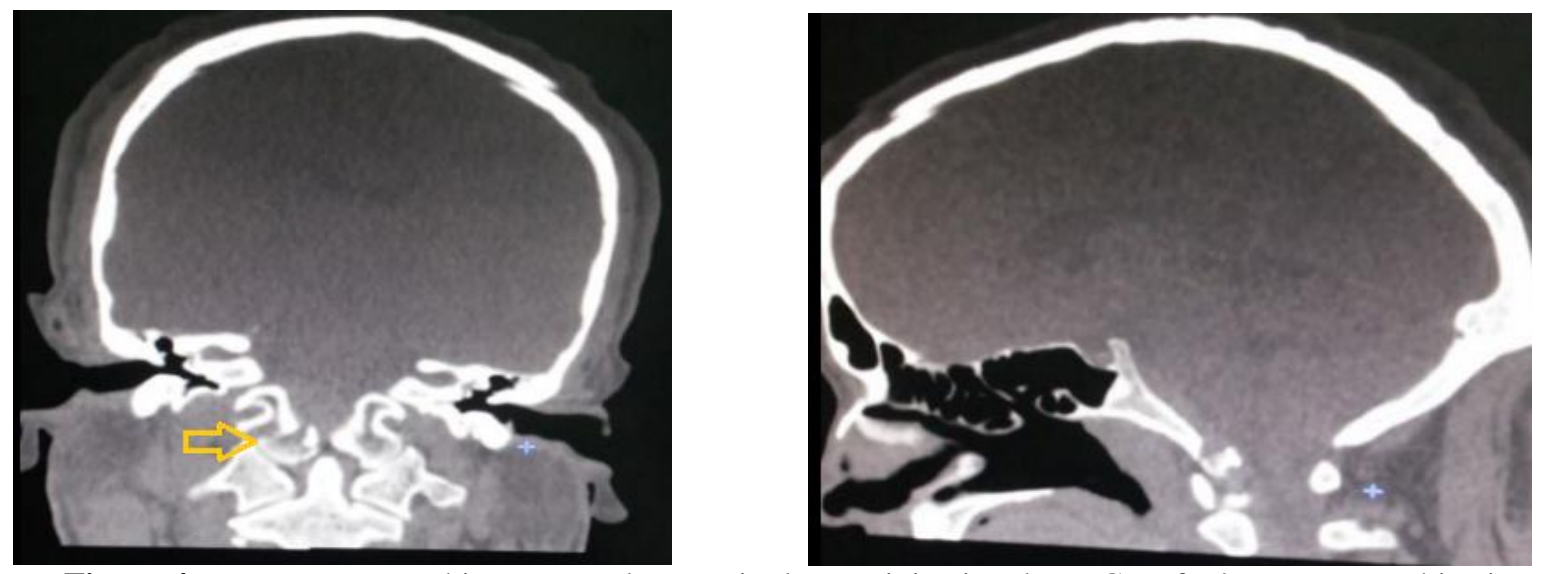

Figure 4:-coupe scanographique coronale et sagittale sans injection de PDC en fenêtre osseuse, objectivant la fracture du clivus étendue au condyle occipital droit.

\section{Référence:-}

1. Tahir MZ, Quadri SA, Hanif S, Javed G. Traumatic retroclival epidural hematoma in pediatric patient: Case report and review of literature. Surg Neurol Int. 2011 Jun;2:78

2. Bernardo Ratilal, MD, Pedro Castanho, MD, Carlos Vara Luiz, MD, Joa o Oliveira Antunes, MD. Traumatic clivus epidural hematoma: case report and review of the literature. Surgical Neurology. 2006 ; 66: 200-202.

3. Shane Tubbs R, Christoph PhD, Griessenauer J, Todd Hankinson MD. Retroclival Epidural Hematomas: A Clinical Series. Neurosurgery. $2010 ; 67: 404-407$.

4. Nguyen H S, Shabani S, Lew S. Isolated traumatic retroclival hematoma: case report and review of literature. Childs Nerv Syst. 2016 ; 32: 1749-1755.

5. Mizushima H, Kobayashi N, Sawabe Y. Epidural hematoma of the clivus. Case report. J Neurosurg 1998 ; 88:590 -3.

6. Ratilal B, Castanho P, Vara Luiz C, Antunes JO. Traumatic clivus epidural hematoma: case report and review of the literature. Surg Neurol. 2006 ; 66(2):200-202.

7. Yang BP. Traumatic retroclival epidural hematoma in a child. Pediatr Neurosurg. 2003;39(6):339-340.

8. Paterakis KN, Karantanas AH, Hadjigeorgiou GM, Anagnostopoulos V, Karavelis A Clin. Retroclival epidural hematoma secondary to a longitudinal clivus fracture. Neurol Neurosurg $2005 ; 108: 67-72$.

9. Kwon TH, Joy H, Park YK, Chung HS. Traumatic retroclival epidural hematoma in a child: case report. Neurol Med Chir (Tokyo) $2008 ; 48: 347-350$. 